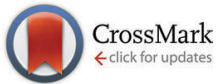

Cite this: Phys. Chem. Chem. Phys., 2016, 18, 21710

Received 27th May 2016 ,

Accepted 7th July 2016

DOI: $10.1039 / c 6 c p 03674 k$

www.rsc.org/pccp

\title{
Fluorescence lifetime imaging of optically levitated aerosol: a technique to quantitatively map the viscosity of suspended aerosol particles $\dagger$
}

\author{
C. Fitzgerald,$\ddagger^{a}$ N. A. Hosny, $\S^{b}$ H. Tong, $\boldsymbol{q}^{a}$ P. C. Seville, ${ }^{c}$ P. J. Gallimore, ${ }^{a}$ \\ N. M. Davidson, ${ }^{d}$ A. Athanasiadis, ${ }^{b}$ S. W. Botchway, ${ }^{e}$ A. D. Ward, ${ }^{e}$ M. Kalberer, ${ }^{a}$ \\ M. K. Kuimova ${ }^{b}$ and F. D. Pope ${ }^{\star d}$
}

\begin{abstract}
We describe a technique to measure the viscosity of stably levitated single micron-sized aerosol particles. Particle levitation allows the aerosol phase to be probed in the absence of potentially artefactcausing surfaces. To achieve this feat, we combined two laser based techniques: optical trapping for aerosol particle levitation, using a counter-propagating laser beam configuration, and fluorescent lifetime imaging microscopy (FLIM) of molecular rotors for the measurement of viscosity within the particle. Unlike other techniques used to measure aerosol particle viscosity, this allows for the nondestructive probing of viscosity of aerosol particles without interference from surfaces. The well-described viscosity of sucrose aerosol, under a range of relative humidity conditions, is used to validate the technique. Furthermore we investigate a pharmaceutically-relevant mixture of sodium chloride and salbutamol sulphate under humidities representative of in vivo drug inhalation. Finally, we provide a methodology for incorporating molecular rotors into already levitated particles, thereby making the FLIM/optical trapping technique applicable to real world aerosol systems, such as atmospheric aerosols and those generated by pharmaceutical inhalers.
\end{abstract}

\section{Introduction}

Aerosol particles are ubiquitous in nature and are widely used and encountered in our day-to-day lives. Their small sizes and correspondingly high surface area to volume ratio provide them with unique chemical and microphysical properties. They play a key role in the interrelated topics of climate and human health. ${ }^{1-5}$

\footnotetext{
${ }^{a}$ Department of Chemistry, University of Cambridge, Lensfield Road, Cambridge, CB2 $1 E W, U K$

${ }^{b}$ Department of Chemistry, Imperial College London, South Kensington Campus, London, SW7 2AZ, UK

${ }^{c}$ School of Pharmacy and Biomedical Sciences, University of Central Lancashire, Preston, Lancashire, PR1 2HE, UK

${ }^{d}$ School of Geography, Earth and Environmental Sciences, University of Birmingham, Edgbaston, Birmingham, B15 2TT, UK. E-mail: f.pope@bham.ac.uk

${ }^{e}$ The Science and Technology Facilities Council, Rutherford Appleton Laboratory, Research Complex at Harwell, Oxfordshire, OX11 OFA, UK

$\dagger$ Electronic supplementary information (ESI) available. See DOI: 10.1039/ c6сp03674k

\# Current address: Biological and Soft Systems, Cavendish Laboratory, 19, J J Thompson Avenue, Cambridge, CB3 0HE, UK.

$\S$ Current address: Tissue Engineering \& Biophotonics, Guy's Campus, Great Maze Pond, London, SE1 9RT, UK.

ศ Current address: Multiphase Chemistry, Hahn-Meitner-Weg 1, B.1.89 55128 Mainz, Germany.
}

To influence the climate, aerosols can scatter and absorb solar and thermal radiation, as well as regulating the cloud characteristics by acting as cloud condensation and ice nuclei. ${ }^{6}$ For health effects, aerosol particles can induce oxidative stress $^{7}$ which has been shown to reduce life expectancy. ${ }^{8}$ However, aerosol particles also provide a crucial tool for the delivery of chemicals to locations which are otherwise hard to access, for example, in the delivery of pharmaceutical drugs to the lungs. ${ }^{9-13}$

The viscosity of aerosol is an important parameter for the atmospheric sciences, materials, food and pharmaceutical industries. For example, heterogeneous chemistry of single atmospheric aerosol particles is controlled by the reactive uptake of trace gases. The viscosity dependent phase states (liquid or solid) can significantly influence the diffusion and ageing process. ${ }^{14-17}$ In addition, viscosity is considered as a key parameter, alongside factors such as particle size and $\mathrm{pH}$, affecting the delivery of nasal drugs and their effective absorption into the body. ${ }^{18-20}$ Medicine delivered by inhalation can be inefficient due to the tendency of the respiratory tract to filter out foreign material by impaction, sedimentation and diffusion. ${ }^{21-24}$ Investigating the water uptake (hygroscopicity) of inhaled aerosolised drugs during transport into the humid airway is important for understanding how they will behave 
during inhalation and this process is expected to be viscositydependent. ${ }^{25}$

There are currently only a limited number of methods available to accurately measure the viscosity of aerosol droplets, due to their small volumes. For example a particle released from a pharmaceutical inhaler has an aerodynamic diameter of 2-5 microns (which is a function of the physical diameter and density), with corresponding volumes on the femtolitre scale. Measurements with commercial instruments such as viscometers and rheometers rely on bulk sample volumes in excess of a few millilitres. Although measurements of bulk samples that constitute the aerosol are possible, these samples will not be truly representative of the particle's chemical complexity and do not allow the environment (such as humidity) of the aerosol to be controlled. ${ }^{26}$

We have developed an imaging technique that uses viscositysensitive fluorescent dyes, termed 'molecular rotors' in conjunction with fluorescent lifetime imaging microscopy (FLIM) that allows for the measurement of the viscosity of aerosol particles, deposited on a microscopic slide, under variable environmental conditions. ${ }^{27,28}$ Use of molecular rotors is an emerging technique that has been previously used in biological applications e.g. to measure diffusion and viscosity in live cells, at a single-cell level. ${ }^{29}$ The main premise of this technique is that an increase in the viscosity of the rotor micro-environment $(\eta)$ will result in an increased fluorescence lifetime, according to eqn (1), which is a rearranged form of the formulae outlined by Förster and Hoffmann. ${ }^{30}$

$$
\tau_{\mathrm{f}}=\frac{z \eta^{\alpha}}{k_{\mathrm{r}}}
$$

Where $\tau_{\mathrm{f}}$ is a fluorescence lifetime, $k_{\mathrm{r}}$ is a radiative decay rate constant, $z$ and $\alpha$ are constants which need to be determined empirically by calibration with known viscosities. ${ }^{29,31,32}$ According to eqn (1), a plot of $\log \tau_{\mathrm{f}}$ as a function of $\log \eta$ will produce a straight line for the viscosity range over which the rotor lifetime is sensitive and can serve as a calibration plot for the rotors. In some cases, the linear relationship breaks down at high viscosities, and a non-linear calibration is required. This is discussed in more detail in the Experimental section. Importantly, since the fluorescence lifetime is concentration independent in the absence of self-quenching, the above method provides a quantitative means of measuring the viscosity in the particle. When the rotor partitions to the whole of the particle, as in this work, then it probes the bulk viscosity. However, if the rotor preferentially partitions to certain regions of the particle then it probes the micro-viscosity of that microscopic region. These micro-viscosity values can be related to the diffusion coefficient of a small molecule (such as the rotor) in such an environment. ${ }^{28,33,34}$ For this work we have selected a hydrophilic rotor and in the aqueous chemical systems studied in this paper, we expect the rotor to partition throughout the whole sample and therefore it will measure the bulk viscosity.

The technique not only provides a viscosity map across the aerosol droplet, but also a means of capturing dynamic changes in viscosity in real-time as the environment of the aerosol changes.
For example, we recently showed that chemical oxidation of organic droplets (ozonolysis of oleic acid) on coverslips leads to changes in the droplet viscosity due to changes in molecular size and functionality. ${ }^{27}$ However, the presence of the surface of the coverslip prevents this method from truly simulating the real aerosol phase and could interfere with important processes such as crystallisation. Thus in the present study, the use of fluorescent molecular rotors is extended to aerosol particles that are optically trapped within a continuous gas flow, thereby avoiding any artefacts associated with contact with surfaces. Optical trapping is a diverse tool used across the physical and biological sciences that allows single particles to be securely suspended in a gas or liquid using laser beams focused by a microscope objective. ${ }^{35-38}$ Previous work successfully combined FLIM with optical trapping for temperature sensing purposes in microfluidic devices. ${ }^{38}$ Here we describe the combination of FLIM of viscosity sensitive molecular rotors with optical trapping of single aerosol particles. This technical advancement allows for viscosity mapping of aerosol particles suspended in a gas, i.e. probing the true aerosol phase, using an optical trapping set-up. The technique is non-destructive and therefore allows multiple measurements to be performed on a single aerosol particle, such as following changes in viscosity overtime as the environment is adjusted. We highlight the robustness of the technique with viscosity measurements of binary sucrose-water aerosols which provide a well-studied standard. We proceed to measure the viscosity of the pharmaceutically important ternary mixture of salbutamol sulphate-sodium chloride-water in a model lung environment. The viscosity values obtained in this work provide information on the rates of diffusion of water within aerosol, partitioning to and from the aerosol phase under different conditions and also relate to the hygroscopic behaviour of material components found within inhaler formulations within the lung, currently unavailable with any other methods.

\section{Experimental}

\section{Molecular rotor viscosity calibration}

For fluorescent lifetime imaging experiments, the viscosity probe 3,3'-diethylthiacarbocyanine iodide (Cy3, 36809, Santa Cruz Biotechnology, 99\% purity) was used. Сy3 is a water soluble dye which is well suited for studying aqueous aerosol particles. In order to convert the fluorescence lifetimes to viscosity values, a calibration plot was obtained, as described previously (Fig. 1) ${ }^{28}$ Briefly, the fluorescence lifetimes of bulk aqueous sucrose solutions of varying sucrose/water ratios and known viscosity, containing Cy3, were measured (Fig. 1A). The non-saturated sucrose/water solutions $(<67 \% \mathrm{w} / \mathrm{w})$ were measured using a Discovery Hybrid (DH) Rheometer (HR-3, TA Instruments) by performing a flow ramp test on each sample. For the higher viscosity solutions ( $>67 \% \mathrm{w} / \mathrm{w})$, direct measurements of viscosity were impossible as sucrose precipitated out. Instead the theoretical model of sucrose by Génotelle et al. was used to calculate the solution viscosity. ${ }^{39}$ The supersaturated solutions were prepared by controlled water evaporation. 

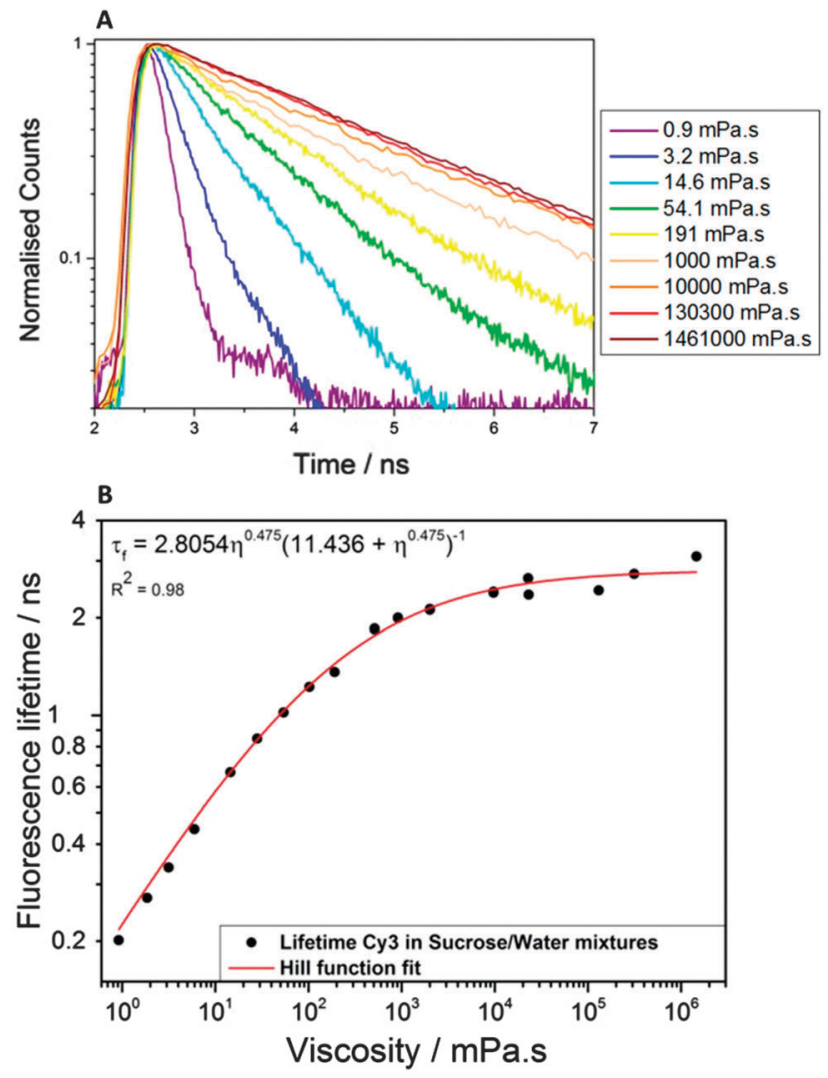

Fig. 1 (A) Example lifetime decays of $\mathrm{Cy} 3$ in sucrose/water solutions of increasing viscosity. (B) Calibration of $\mathrm{Cy} 3$ molecular rotor lifetimes in the sucrose/water solutions of increasing viscosity. Hill formula used to fit data and for conversion of fluorescence lifetimes into viscosity. It should be noted that the sensitivity of the calibration will decrease as lifetimes approach the asymptote.

Solutions of $40 \%(\mathrm{w} / \mathrm{w})$ sucrose/water, containing the Cy3 dye, were heated at $100{ }^{\circ} \mathrm{C}$ under vacuum (150 mbar). The water activity in each sample was determined using a Karl Fischer titrator (Mettler Toledo). The water activity could then be input into Génotelle's parameterisation to calculate the viscosity. We have also previously performed calibration of Cy3 in methanol/ glycerol. ${ }^{40}$ There are small differences in the fitted lifetimes but the general trend is the same. Therefore we believe that for aqueous-based systems the sucrose calibration is suitable.

The lifetime as a function of viscosity could then be plotted to produce the calibration graph shown in Fig. 1B.

In the case of the Cy3 calibration, the lifetime vs. viscosity relationship is linear only between 1-100 $\mathrm{mPa} \mathrm{s}$; at higher viscosities the lifetime response becomes non-linear (Fig. 1B). Therefore, to parameterise the entire viscosity range up to $10^{6} \mathrm{mPa} \mathrm{s}$, a Hill function was used. The non-linearity of the lifetime-viscosity relationship, and the breakdown in the Förster-Hoffmann equation, is discussed elsewhere. ${ }^{41}$ For the measurements discussed here, this means that in the range 2-2.8 ns, there is decreasing sensitivity of the rotor's fluorescence lifetime to viscosity. This is due to the radiative relaxation of the excited state dominating over non-radiative processes at higher viscosities. ${ }^{29}$ For Cy3, lifetimes longer than 2.8 ns are not convertible into viscosities. This plateau effect means that any small differences in fluorescence lifetimes in this range, for example due to variability between repeated measurements, error in the lifetime fit or general spread in fluorescence lifetimes across a FLIM image, will correspond to a broad viscosity range, spanning $<100 \mathrm{~s}$ of $\mathrm{Pa}$ s. Therefore it is recommended that viscosities converted from lifetime measurements $>2 \mathrm{~ns}$ are taken as an approximation of the order of magnitude of the viscosity rather than an absolute viscosity value.

Stock solutions of the Cy3 dye were prepared at a concentration of $1 \mathrm{mM}$ in DMSO ( $\geq 99.5 \%$, Sigma Aldrich). This stock was diluted in MilliQ water $(18.5 \mathrm{Ohm})$ containing the solutes of interest, to achieve the dye concentration 50-190 $\mu \mathrm{M}$. We demonstrated that at these concentrations the lifetime of $\mathrm{Cy} 3$ in bulk solution is not affected by aggregation or quenching of the dye (described in the ESI $\dagger$ ).

Sucrose ( $\geq 99.5 \%$, GC standard, Sigma Aldrich), $\mathrm{NaCl}$ ( $\geq 99.5 \%$, Sigma Aldrich) and salbutamol sulphate (99\%, Alfa Aesar) were used as provided with no further purification. Sucrose solutions for aerosol preparation were $0.75 \mathrm{M}$. The mixed $\mathrm{NaCl}$ and salbutamol sulphate solutions were prepared at concentrations of $\mathrm{NaCl}$ and salbutamol sulphate of $1.35 \mathrm{M}$ and $0.1 \mathrm{M}$, respectively. This equates to a weight to weight (w/w) ratio of $1.4: 1$. This is comparable to ratios that are found commercially in medical inhalers, for example, the commercially available Ventolin ${ }^{\circledR}$ Respirator Solution, which contains $0.5 \% \mathrm{w} / \mathrm{v}$ salbutamol sulphate. $1 \mathrm{ml}$ of this solution (containing $5 \mathrm{mg}$ salbutamol sulphate) is used with $1.5 \mathrm{ml} 0.9 \% \mathrm{NaCl}(13.5 \mathrm{mg}$ $\mathrm{NaCl}$ ), resulting in a $\mathrm{NaCl}$ : salbutamol sulphate ratio of $2.7: 1 .^{42}$ To test the applicability of the set-up to analyse real-world aerosol, the combined trapping/FLIM set-up was used to trap aerosol directly generated from a pressurized metered dose inhaler (pMDI) (Salamol ${ }^{\mathrm{TM}}$, IVEX, 100 microgram dose Salbutamol Sulphate) which is used to treat asthma in humans.

\section{Experimental set-up: optical trapping and FLIM}

The experimental set-up combines an optical trap with confocal laser scanning microscopy and fluorescence lifetime acquisition. This allows for liquid and solid particles to be suspended in air thereby eliminating any possible artefacts caused by surfaces. The optical trap uses an asymmetric counter propagating dual beam (CPDB) geometry, ${ }^{22,36,43}$ combined with single photon excitation and fluorescence lifetime acquisition using TimeCorrelated Single Photon Counting (TCSPC).

Fig. 2 shows the optical beam arrangement for the asymmetric counter-propagating trap configuration built around an inverted microscope (Nikon Eclipse TE2000-U or a Ti-E), combined with single-photon (SP) FLIM (see below). The optical trapping beam was a split $1064 \mathrm{~nm}$ (Ventus, Laser Quantum) continuous wave light source. Stable trapping powers for the aerosol for the upward and downward beam powers were in the range of 4-9 $\mathrm{mW}$ (upward beam) and 5-14 $\mathrm{mW}$ (downward beam). After the beam expansion optics the final beam width was $8 \mathrm{~mm}$, enough to slightly overfill the back aperture of the lower objective (water-immersion, $63 \times$, NA 1.2, Leica Microsystems). The top trapping beam was $4 \mathrm{~mm}$ in diameter and 


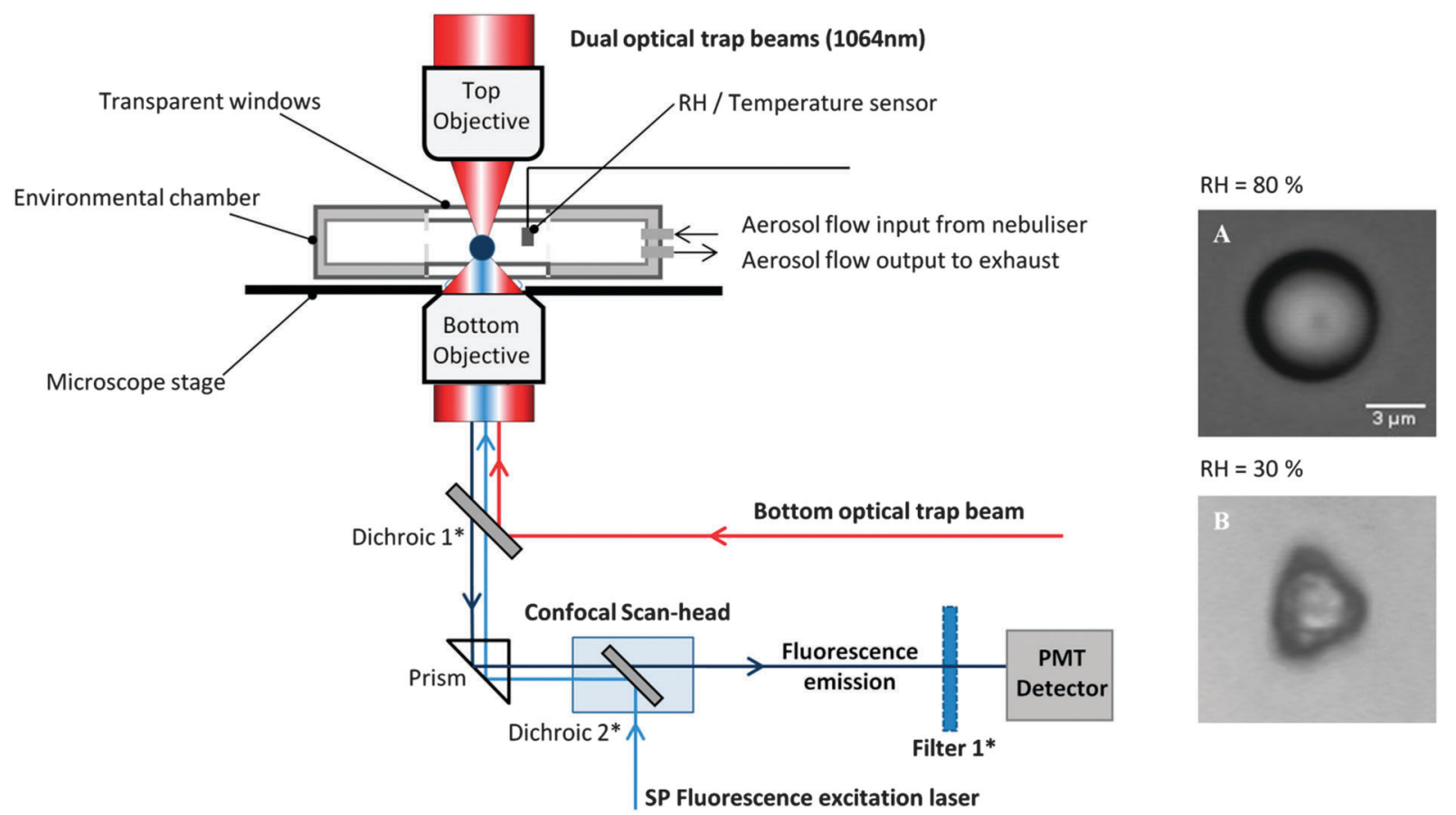

Fig. 2 Schematic diagram of the optical trapping and FLIM arrangement for the single photon (SP) FLIM set-up, including view of the humidity controlled sample chamber. Dichroic 1* (Chroma Zt1064rdc): reflects $1064 \mathrm{~nm}$ laser and transmits below $940 \mathrm{~nm}$. Dichroic 2* (Chroma Technologies DC/zt405488-543-700-950 rpc): reflects SP fluorescence excitation 470-495 nm. Filter 1*: blocks $490 \mathrm{~nm}$ SP fluorescence excitation (LP $530 \mathrm{~nm}$, Thorlabs) in SP set-up using the supercontinuum white light laser source. Images (A) and (B) are brightfield images of an aqueous suspended droplet and a crystal of $\mathrm{NaCl}+$ aqueous salbutamol sulphate respectively.

focused using a 50× objective (Mitutoyo Plan Apo NIR, 50×, NA 0.42 LWD).

The use of a counter propagating trap allows for the stable trapping of non-spherical particles, compared to other configurations. $^{36}$ The size range of the trapped particles was between 2 and $12 \mu \mathrm{m}$. To demonstrate the stability of the optical trap, we securely held both spherical and irregular particles for at least 4 hours within the optical trap at room temperature. Fig. 2A is a brightfield image of an aqueous suspended $\mathrm{NaCl}+$ salbutamol sulphate droplet at a relative humidity $(\mathrm{RH})$ of $80 \%$. Fig. $2 \mathrm{~B}$ is an image of a suspended crystal of $\mathrm{NaCl}+$ aqueous salbutamol sulphate that formed when the $\mathrm{RH}$ was dropped from 80 to $30 \% \mathrm{RH}$. This image clearly shows that non-spherical particles can be trapped with this optical set-up. This stable optical trapping opens up the possibility of measuring particles with a range of phases and morphologies, as well as following the transition of a spherical aqueous droplet to a non-spherical crystalline particle without losing the particle from the trap.

For SP fluorescence excitation, either a 40 ps pulsed $473 \mathrm{~nm}$ laser (BDL-473-SMC Becker and Hickl GmbH, $80 \mathrm{MHz}$ ) was used or a supercontinuum white light laser (SuperK COMPACT, NKT Photonics), with a medium pinhole setting on the confocal scan-head (Nikon EC2) to block out of plane fluorescence from the PMT (Hamamatsu R3809U). A modified confocal scan head was used to enable observation of both fluorescence intensity and fluorescence lifetime images. ${ }^{44}$ Fluorescence emission generated by the SP excitation (SPE) set-up was detected using a long pass filter 530LP (Thorlabs, GmBH). A custom-built stainless steel humidity-controlled chamber (of approximate $80 \times 80 \times 15 \mathrm{~mm}$ dimensions) was used for optical trapping with the laser foci adjusted to be close to the lower window. It is appreciated that Mie scattering and resonance of illumination light within a spherical droplet could potentially cause variation in the absolute illumination intensity at any given point. However, the presence of "resonance structure" in laser illumination of the droplet, if it occurred, would not alter the fluorescence lifetime decay that is measured. The cavity quality of micron scale droplets is sufficiently low $(Q=6000$ for similar size droplets) that we do not expect, or observe, ring down effects in the droplet cavity $(<1 \mathrm{ps}) .{ }^{58,59}$ Hence the fluorescence lifetime decay (ns) is not altered.

Circular glass coverslips (VWR, thickness $0.133 \mathrm{~mm}$, $\varnothing 35 \mathrm{~mm}$ ), sealed onto the top and bottom of the environmental chamber, allowed the trapping and FLIM laser beams to enter the cell.

The chamber, connective tubing and nebuliser were washed with soap, water and methanol between experiments. Droplets were generated using an ultrasonic nebuliser (Schill Medical, Aerosonic mobil), and droplets were delivered into the environmental chamber in the microscope stage via $4 \mathrm{~mm}$ (inner diameter) Teflon tubing. After a particle was trapped, a constant flow of $200 \mathrm{ml} \mathrm{m^{-1 }}$ of humidified nitrogen was passed through the chamber, while FLIM measurements were taken. The RH within the chamber was controlled (between 0-95\%) by varying the flow rates of dry nitrogen and water-saturated 

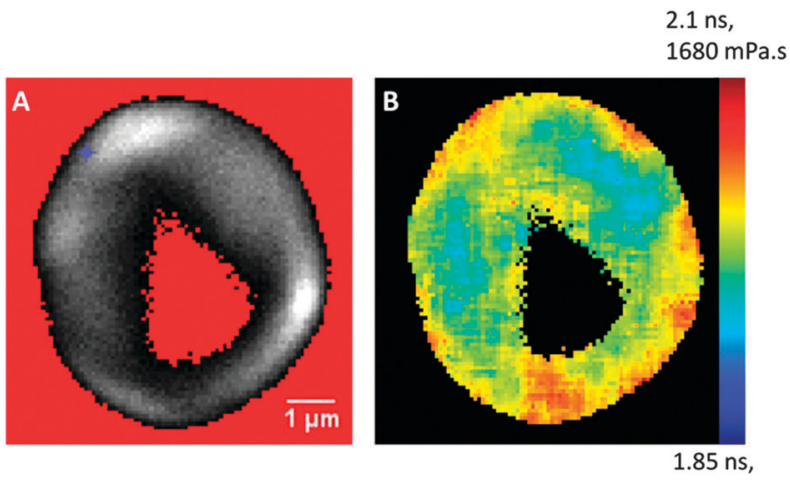

$680 \mathrm{mPa} . \mathrm{s}$

Fig. 3 Fluorescence intensity (A) and FLIM (B) images of a salbutamol sulphate/ $\mathrm{NaCl}$ aerosol trapped at $35 \% \mathrm{RH}$. The $\mathrm{NaCl}$ fraction has crystallized with traces of $\mathrm{Cy} 3$ dye remaining above and below the crystal in the aqueous salbutamol sulphate fraction. This fluorescence intensity has been thresholded from the image to highlight the crystals position. The colour scale represents the distribution of lifetimes in the FLIM image. The average lifetime $\left(\tau_{\mathrm{av}}\right)$ and viscosity measured for this particle was $1.99 \pm 0.031 \mathrm{~ns}$ and $1240 \pm 130 \mathrm{mPa}$ s respectively. Estimation of the variability in the measured values is discussed in the ESI. $\dagger$

nitrogen generated using a water bubbler. The $\mathrm{RH}$ and temperature were constantly monitored using a digital humidity sensor placed inside the chamber (SHT-75, Sensirion). Opaque perspex housing enclosed the whole microscope setup to reduce light contamination. Fluorescence intensity images were acquired using the confocal software EZ-C1 for Nikon (C1/C2 confocal, Gold version 3.90). Fluorescence lifetime images were obtained using the Becker and Hickl SPC-830 or SPC-150 TCSPC PCI card and SPCM acquisition software version 9 (pixel grid of $128 \times 128$ and ADC resolution of 256). The instrument response function (IRF) was recorded using a scattering solution of colloidal silica (Ludox). An example fluorescence intensity and FLIM image of a partially crystalline salbutamol sulphate/ $\mathrm{NaCl}$ particle trapped at $35 \% \mathrm{RH}$ is shown in Fig. 3, demonstrating the imaging capability of the set-up. The structure from the $\mathrm{NaCl}$ crystal can be seen in the centre of the trapped aerosol. Any fluorescence intensity in the centre of the particle originates from the salbutamol sulphate fraction above and below the crystal, since upon crystallisation the dye would have been lost from the $\mathrm{NaCl}$ fraction. Therefore in image A the intensity in the centre has been removed from the image to clearly highlight where the crystal is located.

It was found that long exposures to the FLIM laser, at constant temperature and $\mathrm{RH}$ environment, resulted in some lengthening of the measured fluorescence lifetime, discussed further in the ESI. $\dagger$ The exact mechanism of this fluorescence lifetime lengthening is unknown, but care was taken to minimize the effect by turning off the FLIM laser in between measurements on the same particle and reducing the laser power to the minimum possible.

\section{Time-resolved data analysis}

In order to determine the fluorescence lifetime, images were exported to and analysed using either the TRI2 (Version 2.7.6.1,
Gray Institute for Radiation Oncology and Biology ${ }^{45}$ or FLIMfit (OMERO) software. The time resolved decays of Cy3 were previously shown to conform to a biexponential decay. ${ }^{27}$ Decays for each pixel were then fit using the Levenberg-Marquardt algorithm. ${ }^{46}$

The average lifetimes were calculated using eqn (2) and converted to viscosities using the calibration curve (Fig. 1B).

$$
\tau_{\mathrm{av}}=\frac{\sum_{i} A_{i} \tau_{i}^{2}}{\sum_{i} A_{i} \tau_{i}}
$$

Where $A_{i}$ is the intensity of component $\tau_{i}$ as contributes to the overall lifetime, $\tau_{i}$ is the component lifetimes, $\tau_{\mathrm{av}}$ is the biexponential fluorescence lifetime.

FLIM images of the particles were created using fluorescence lifetimes in each pixel, with appropriate binning, mapped across the image. This binning involves grouping together and averaging the time-resolved signal from multiple pixels so that at least 1000 counts at the peak maximum were reached for each. This is to obtain a fit with an acceptable degree of accuracy for a biexponential decay. ${ }^{27,45,47,48}$ Pixel sizes ranged from $0.8 \mu \mathrm{m}^{2}$ up to binning the whole image to gain a single average lifetime, depending on the fluorescence intensity. The intensity of an image, and therefore the binning required, will depend on factors such as the dye concentration and the viscosity of the sample. The higher the viscosity the greater the fluorescence intensity, as shown by the relationship between viscosity and intensity defined by Förster and Hoffmann. ${ }^{30}$ Viscosity was varied in this work by changing the $\mathrm{RH}$, i.e. the lower the $\mathrm{RH}$, the greater the viscosity of the sample, due to loss of water from the liquid droplet to the surrounding atmosphere. Data was only used in an image if the fit of the experimental data to a biexponential curve had a reduced chi-squared in the range $0.8>\chi_{\mathrm{r}}^{2}<1.2$.

\section{Results and discussion}

\section{Calibration of optical trapping and FLIM: sucrose as model system}

Sucrose is commonly used as a surrogate to model features of organic atmospheric aerosol. The water content and thus viscosity of binary sucrose/water particles is dependent on the RH. ${ }^{27,49-51}$ A series of aqueous sucrose aerosol measurements were carried out to ensure that upon successful trapping of particles, it was possible to accurately measure their viscosity over a range of RHs. Sucrose/water was considered to be a suitable system to check that the trapping/FLIM system was measuring accurate viscosities firstly because there are no expected phase changes in the $\mathrm{RH}$ range measured that will be significantly affected by slide contact for example by heterogeneous nucleation. Secondly because the only diffusing species is water, it was assumed that the presence of the cover-slide would have minimal influence on the final viscosity of the sucrose droplets when given sufficient time to equilibrate. 

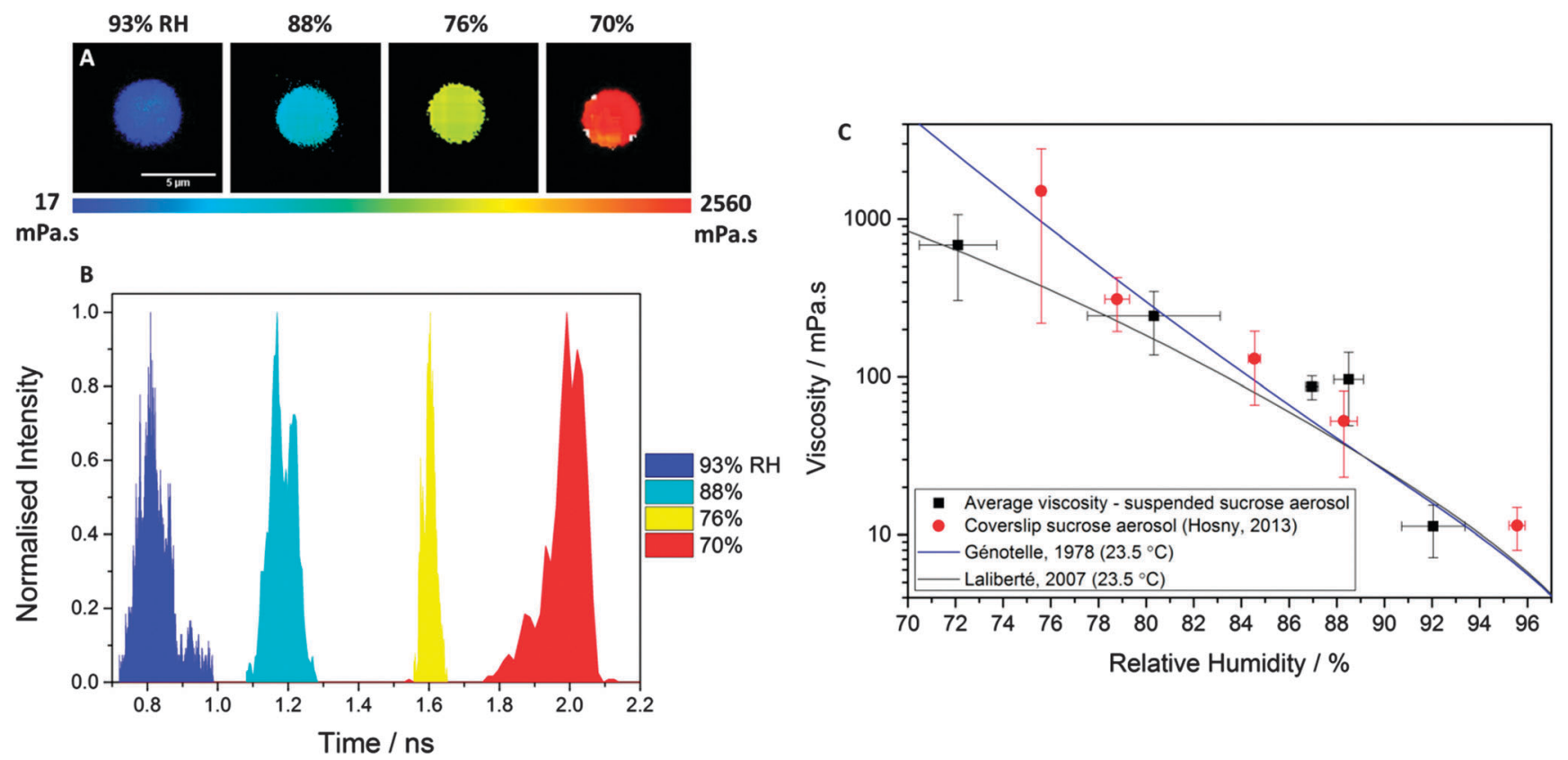

Fig. 4 (A) Example FLIM images for sucrose/Cy3 particles at different RHs (93, 87, 76 and 70\%). (B) Fluorescence lifetime histograms for each image, representing the spread in the lifetimes across the image. An arbitrary colour scale has been assigned to the histograms and the images to label lifetime. (C) Change in viscosity of sucrose with $\mathrm{RH}$. Black squares: average change in viscosity with $\mathrm{RH}$ for suspended sucrose/Cy3 aerosol \pm standard deviation. Red circles: average change in viscosity with $\mathrm{RH}$ for coverslip sucrose/Cy3 droplets \pm standard deviation. ${ }^{27}$ Grey line: calculated change in sucrose viscosity with RH using Lalibertés sucrose viscosity parameterisation. Blue line: calculated change in sucrose viscosity with RH using Génotelle's empirical equation for sucrose viscosity as a function of water content. Sucrose models set at $23.5^{\circ} \mathrm{C}$

The image panel (Fig. 4A) shows the FLIM imaging capability of the set-up. FLIM images of four aqueous sucrose droplets held at different RHs are shown, alongside their corresponding fluorescence lifetime histograms, representing the distribution in lifetimes across the image (Fig. 4B). In the images at 93 and $88 \% \mathrm{RH}$, the binning gives a spatial resolution of approximately $0.8 \mu \mathrm{m}^{2}$; for images at 76 and $70 \% \mathrm{RH}$, greater binning was required to obtain sufficient counts for analysis. The dye was homogeneously spread throughout the droplets. A false rainbow colour scale has been assigned to the droplets to label the lifetimes. The histogram and decay curves clearly show the shift to longer lifetimes as the $\mathrm{RH}$ is decreased from 93 to $70 \% \mathrm{RH}$, indicating an increase in viscosity. Fig. $4 \mathrm{C}$ shows the fluorescence lifetimes converted into viscosities (using the calibration curve, Fig. 1B) for a selection of aqueous sucrose aerosol droplets suspended in the optical trap. FLIM measurements were performed for droplets held at a constant $\mathrm{RH}$ and $\sim 10$ droplets were measured for each RH. This data was compared to our previously published data set recorded for particles deposited on a cover slip. ${ }^{27}$ As expected, the fluorescence lifetime decreases following an increase in $\mathrm{RH}$, as water is taken up by the aerosol, and its viscosity decreases. The trapping data agrees very well (within the spread of the data) with the coverslip work. The data has been compared to two literature parameterisations for the viscosity of sucrose with varying water content, Laliberte $(2007)^{52}$ and Génotelle (1978) ${ }^{39}$ and again show good agreement. Laliberté (2007) published a model for calculating the viscosity of 74 different aqueous solutions over a range of temperatures and concentrations. This study developed parameters using over 9000 data points from previous studies that looked into the viscosity of one solute in water. The model was validated by comparing the calculated values for more than one solute in a system to published experimental values. Secondly, the simple empirical equation using the mole fraction of the sugar (7) by Génotelle (1978) as outlined by Longinotti (2008), was used (further details of these literature parameterisations and the relevant equations are discussed in the ESI $\dagger$ ). The presence of the optical trapping laser resulted in a slight heating effect, which had a negligible effect on the viscosity, (discussed further in the ESI $\dagger$ ). From these measurements, we have demonstrated the use of the FLIM/optical trapping technique to accurately measure viscosity of sucrose aerosol in the $\mathrm{RH}$ range greater than $70 \%$.

\section{Hygroscopicity of salbutamol sulphate/ $\mathrm{NaCl}$ aerosol}

It is not only atmospheric particles that are affected by RH; the same is true for inhaled pharmaceutical drugs. The size and water content of these pharmaceutical particles are dependent upon the environmental RH. Size determines the location of particle deposition within the respiratory tract and water content determines the phase, viscosity and diffusivity of the particle. The effectiveness of the drug delivery to the patient after deposition are in part dependent upon the ability of the drug to dissolve in fluids present on, and be absorbed across, the respiratory epithelia before being removed by mechanisms such as phagocytosis by respiratory macrophages, degradation by lung enzymatic action or elimination via the mucociliary escalator. 

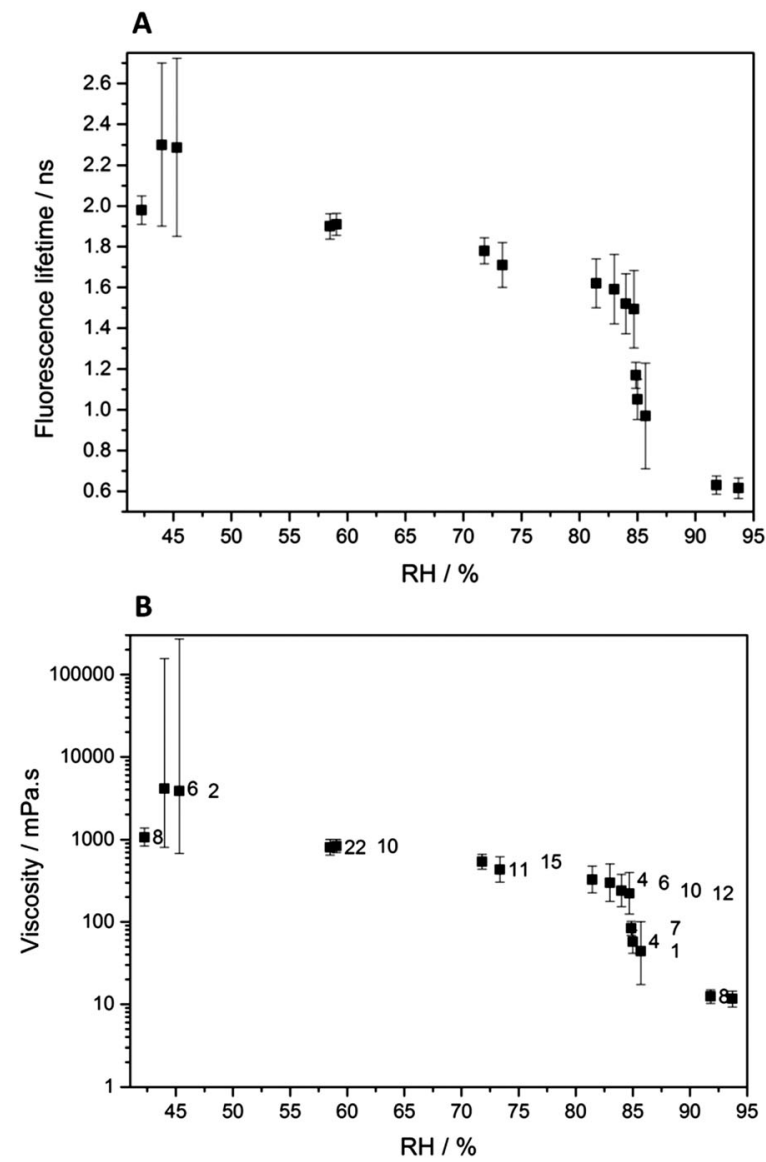

Fig. 5 (A) Fluorescence lifetimes (ns) of salbutamol sulphate/ $\mathrm{NaCl} / \mathrm{Cy} 3$ aerosol at different RHs. (B) Lifetimes converted into viscosity (mPa s). Black number labels are the equilibration times (minutes) at each $\mathrm{RH}$. Error bars are the standard deviations of the spread of the lifetimes within each FLIM image.

Thus a detailed knowledge of the water uptake (and its kinetics) is needed to improve inhaler drug delivery strategies. ${ }^{22,53}$

The bronchodilator salbutamol sulphate, diluted with $0.9 \%$ $\mathrm{w} / \mathrm{v} \mathrm{NaCl}$, is commonly used in nebulisers for the treatment of asthma, particularly for acute-asthmatic attacks. ${ }^{54}$ Viscosity measurements of the trapped and equilibrated salbutamol sulphate/ $\mathrm{NaCl}$ aerosol droplets following a step wise change in RH are shown in Fig. 5. Our data shows that the lifetimes (Fig. 5A) and, consequently, viscosity (Fig. 5B) of these aerosols change between 10 to $10^{3}-10^{5} \mathrm{mPa} \mathrm{s}$ in the $\mathrm{RH}$ range from $45-93 \%$. These viscosities correspond to a mixing timescale for water (radius $1 \AA$ ) of $\sim 100-0.003$ seconds respectively, assuming a Stokes-Einstein relationship for a $5 \mu \mathrm{m}$ diameter droplet, i.e. the inhalable size fraction is $2-5 \mu \mathrm{m}^{22}$ (aerodynamic diameter), and using the upper limit viscosity of $\sim 10^{5} \mathrm{mPa} \mathrm{s},{ }^{55}$ (details of the estimation of mixing times are found in the ESI $\dagger$ ). These estimates suggest that the experimental equilibration times (several minutes per point) were sufficient for the droplets to reach their maximum viscosity at all investigated RHs. Since the approximate timescale for inhalation and particle impaction is $\sim 10 \mathrm{~s}$, the water mixing time estimate implies that the aerosol in the inhalable size fraction $(2-5 \mu \mathrm{m})^{22}$ will be at equilibrium with the high RH encountered within the lung. ${ }^{22}$ Slowed diffusion of the active ingredient can be beneficial to slow the release of the drug into the system, reducing the frequency with which the drug must be inhaled. ${ }^{56}$

The viscosity values can be used to estimate diffusion coefficients for water by using the Stokes-Einstein relationship (as described in the ESI $\dagger$ ). Considering that our values are below the glass transition, where the Stokes-Einstein relationship has been shown to break down, this relationship can be applied.

A limited set of comparative coverslip measurements of salbutamol sulphate/ $\mathrm{NaCl} / \mathrm{Cy} 3$ droplets that used the same solution concentrations as the optical trap experiments were carried out. These measurements, in contrast to the optical trap measurements, showed evidence of phase separation, at $\mathrm{RH}>69 \%$, which led us to believe the contact between the coverslip and droplet was influencing the spatial distribution of the solutes in the droplet and therefore would not provide us with reliable lifetimes. Example FLIM images of the observed phase separation are found in the ESI. $\dagger$ Previous FLIM measurements on $\mathrm{NaCl}$ droplets showed premature crystallisation due to the presence of the coverslip surface. ${ }^{27}$ These two artefact causing properties of the coverslip provide justification of the optical trapping technique, which eliminates the coverslip requirement, for the study of multi-component aerosol.

\section{Measuring changes in viscosity due to a humidity perturbation}

We demonstrate the use of the FLIM/optical trapping technique to successfully follow the kinetics of viscosity changes caused by environmental perturbations by increasing the $\mathrm{RH}$ surrounding trapped salbutamol sulphate/ $\mathrm{NaCl} / \mathrm{Cy} 3$ aerosol and measuring the viscosity over time. Given that there is a large and near step-wise increase in humidity (ambient-99\% RH) ) $^{57}$ during the migration of inhaler particles from inhaler to respiratory tract, understanding the behaviour of these particles during such $\mathrm{RH}$ changes can be beneficial to understand the process and the mechanism by which aerosol medicines are delivered into the lung, and in particular to predict the site of deposition within the lung.

Fig. 6 shows an example RH increase from 34 to $74 \%$. This situation is close to a scenario when, upon inhalation, particles are subjected to rapid $(\sim 10 \mathrm{~s})$ changes in $\mathrm{RH}$, from ambient to near water-saturation $(\sim 99 \%)$ deep in the airways. Our data (Fig. 6) clearly demonstrate that we measure the expected decrease in viscosity as water is taken up by the particle. The measured end-point viscosity of $130 \mathrm{mPa}$ s agrees with the equilibrated aerosol measurements in Fig. 5 for $75 \%$ RH. This is expected since the timescale of the experiment is much longer than the water diffusion timescales calculated for the salbutamol sulphate $/ \mathrm{NaCl}$ aerosol over all RHs.

Although these results will have high relevance to solutionbased inhaler systems, in order to extrapolate these results to understand how real powder and pMDI (pressurised metereddose inhaler) particles will respond to increases in $\mathrm{RH}$, the particles' RH trajectory must be considered, as this will affect the rate at which the particles take up water during inhalation. 


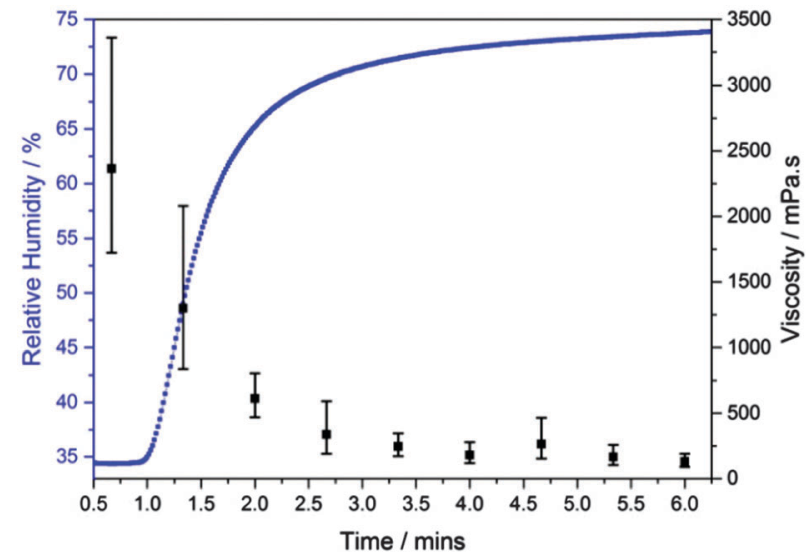

Fig. 6 Change in viscosity for a single salbutamol sulphate/ $\mathrm{NaCl} / \mathrm{Cy} 3$ aerosol (black squares) during an increase in $\mathrm{RH}$ from 34 to $74 \%$ (blue line). Error bars are the standard deviations of the spread of the lifetimes within each FLIM image.

The droplets used in these experiments were nebulised from an aqueous solution. Therefore, once dried out to lower RHs, they will still contain some traces of water, allowing water to be taken up more readily when rehydrated. Whereas pMDI inhaler particles start off dry. Therefore dry generated aerosol will likely show more complex behaviour during inhalation, especially when RH mediated phase changes occur, such as deliquescence. Some delay could therefore be expected in equilibration for real inhaler aerosol.

\section{Doping of trapped aerosol}

In the previous examples of trapped particles (Fig. 2-6), fluorescent molecular rotors were incorporated into solutions of model aerosol components that were later nebulised, thus the trapped aerosol droplets already contained the fluorescent probe from the start. However, this strategy is not always applicable, for example, when the viscosity of particles generated by medical inhalers is studied. To enable this technique to investigate such 'real world' aerosol systems, in which molecular rotors are absent, a new experimental strategy was developed to incorporate molecular rotors into particles subsequent to their capture in the optical trap. Firstly, we show that it is possible to trap particles directly nebulised from a medical inhaler, akin to that demonstrated by Tong et al. (2014). ${ }^{22}$ These particles do not initially contain a molecular rotor to allow FLIM measurements. However the molecular rotors are then incorporated into the trapped aerosol particle through coagulation with molecular rotor-containing aerosol particles. These smaller particles are generated using a nebuliser subsequent to trapping of the target aerosol particle. It was found that trapping directly from the inhaler required very careful alignment of the optical trapping lasers and therefore in future experiments, in order to achieve a greater range of $\mathrm{RH}$ measurements over a longer time period, some optimisation is required and this experiment is a demonstration of the proof-of-principle.

A pMDI inhaler particle was trapped by injecting it directly into the chamber. Before addition of the dye, the aerosol

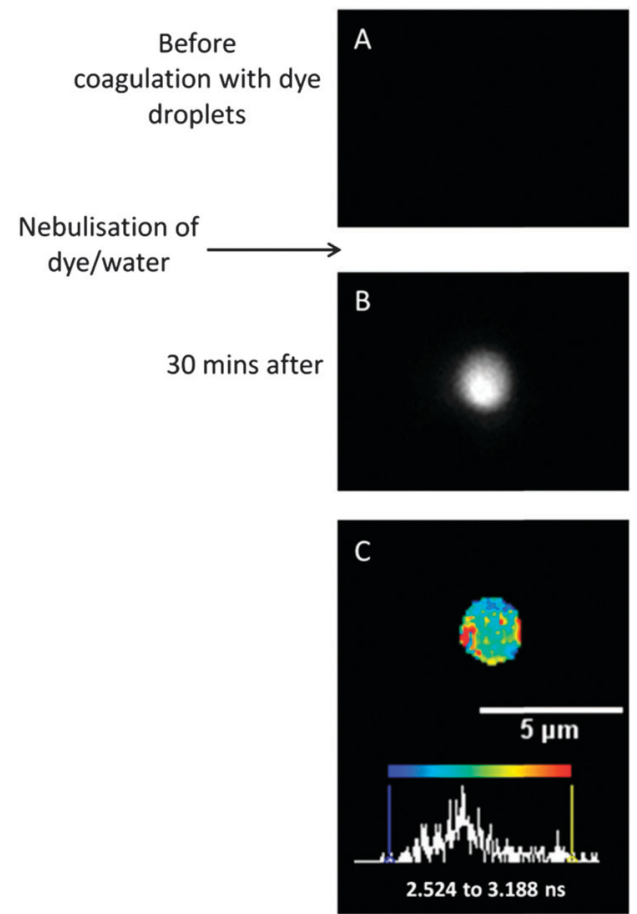

Fig. 7 Imaging of a suspended medical inhaler particle equilibrated at $48 \% \mathrm{RH}$, before $(\mathrm{A})$ and after ( $\mathrm{B}$ and $\mathrm{C}$ ) collision with nebulised aqueous dye aerosol. Image panels correspond to (A and B) confocal (fluorescence intensity), (C) fluorescence lifetime distribution. A colour scale corresponds to the lifetime.

particle exhibited no fluorescence signal, as shown by the fluorescence intensity image (Fig. 7A). The molecular rotor could then be introduced. Aerosol containing the molecular rotor is generated from an aqueous solution of Cy3 $(100 \mu \mathrm{M})$. Whilst we were unable to measure the size of this aerosol, it is known that they will be much smaller in size, compared to the trapped particle, because of the low concentration of solute used to stabilise the droplet in the sub-saturated conditions of the environmental chamber. The dye-containing aerosol was introduced into the chamber until several collisions with the trapped inhaler particle were observed on the brightfield image. This only required a short $(\sim 5 \mathrm{~s})$ period of nebulising the dye aerosol. A small amount of water will have been introduced into the trapped particle matrix during nebulisation. However the particle was then allowed to equilibrate at $48 \%$ RH for 30 minutes, which is sufficient time for the particle to re-equilibrate with the surrounding $\mathrm{RH}$ of the chamber. Upon impaction of the dyecontaining droplets, the fluorescence intensity of the trapped inhaler droplet increases significantly, indicating successful collision and efficient dye transfer into the aerosol of interest (Fig. 7B). The dye transfers throughout the particle and not just to the surface. It is noted that the experiment images a slice through the centre of the particle, and although there is a limit to how thin this slice can be due to the resolution limitation enforced by the diffraction limit of light, the thickness limit is on the order of several hundreds of $\mathrm{nm}$ whereas the studied particles are in the size range of several microns. 
Once the particle of interest is doped then FLIM is possible (Fig. 7C), as detailed in the previous sections. An average fluorescence lifetime of $2.8 \pm 0.13 \mathrm{~ns}$ was measured. A large standard deviation (i.e. range) in lifetimes/viscosities are expected for measurements where the average lifetimes measured are $>2 \mathrm{~ns}$. This is due to the loss of sensitivity in the Cy3 calibration plot in this range, as discussed above. The average lifetime is just above our calibration limit for the Cy3 dye shown in Fig. 1B. Therefore a lower limit for the viscosity of the particle can be estimated at $10^{6} \mathrm{mPa}$, indicating that the particle is in a semi-solid to solid state at this $\mathrm{RH}^{22}$ Although there is very little restriction on the composition of the particles that can be probed with this doping technique (inorganic, organic, mixed multi-component particles) due to the variety of dyes available with different solubilities, this experiment highlights that ideally the particles will be initially in a semisolid to liquid state to allow rapid diffusion of the dye throughout the particle on timescales relevant to experiments.

\section{Conclusions}

In this study we describe a new method, FLIM plus optical trapping, for directly measuring the viscosity of levitated aerosol particles, using FLIM of viscosity sensitive fluorescent dyes called molecular rotors. The high stability of the asymmetric counter propagating optical trap allows trapping aerosol particles of 2-12 $\mu \mathrm{m}$ diameter, independent of their chemical compositions and morphologies. The trapping takes place in an enclosed chamber in which the environment can be modified to the required conditions, such as to high RHs that are pharmaceutically relevant for aerosolised drug delivery. We believe that the reported experimental setup represents a significant advance in the study of aerosol viscosity in a non-destructive manner, since suspension of the aerosol removes any potential for interference with the coverslip and avoiding artefacts such as for example, droplet crystallisation at low $\mathrm{RH}$ or phase separation due to different wetting angles of phases upon the contact with the coverslip.

This study used the Cy3 molecular rotor that is sensitive to viscosity changes in polar systems up to $10^{5} \mathrm{mPa}$ s. However, the FLIM/optical trapping technique is not limited to this particular compound and currently studies in our laboratories are underway to investigate non-polar atmospherically relevant aerosols with higher viscosities, incorporating hydrophobic molecular rotors. The response time of the fluorescent rotor Cy3 to changes in viscosity is limited only by the photon counting acquisition time. Hence this combination of the optical trapping and FLIM can be used to probe viscosity changes within a single particle as it responds to changes in the surrounding environment.

We demonstrate the capabilities of this technique by investigating two aerosol systems: a model binary system sucrose/water and a pharmaceutically relevant ternary system, salbutamol sulphate/ $\mathrm{NaCl} /$ water. The particle viscosities of the two systems were measured over a range of RHs. In the latter case it was possible to observe the hygroscopic behaviour of the particles using FLIM following a change in RH. For more viscous aerosol, continuous lifetime measurements will be useful to estimate equilibration times to changes in environment, since the timescales of equilibration will be reduced significantly due to slowed diffusion.

As demonstrated by the pMDI experiment, this technique opens up exciting new possibilities for experiments that will benefit from using suspended particles, such as in the fields of atmospheric, pharmaceutical and material science. Future atmospheric experiments will involve varying the gas phase composition, as well as the $\mathrm{RH}$, surrounding the trapped aerosol to investigate the effect of common reactions in the atmosphere on the aerosol viscosity, such as oxidation by ozone and hydroxyl radicals. These are dominant reactive species that contribute to organic aerosol chemical ageing. Efforts are underway in our laboratories to improve aspects such as the acquisition time required for a measurement so that dynamic processes, such as inhalation for the purposes of investigating aerosolised drug delivery, can be more accurately simulated. The use of brighter dyes will help decrease the acquisition time needed per measurement and also allow the technique to probe spatial approaches closer to the diffraction limit.

In conclusion, our new method offers a new analytical tool for probing the physical and chemical properties of suspended aerosol droplets, without the associated perturbation effects of support surfaces, following dynamic changes in conditions which we believe will prove invaluable for atmospheric and pharmaceutical aerosol studies.

\section{Acknowledgements}

This project was supported financially by the European Research Council, grant 279405 and the Science and Technology Facilities Council (STFC), Central Laser Facility, Programme Access Grant (LSF1207). MKK is thankful to the EPSRC for the Career Acceleration Fellowship (EP/I003983/1). CF acknowledges the Natural Environmental Research Council (NERC) for the personal studentship (NE/J500070/1).

\section{References}

1 D. Dockery, Annu. Rev. Public Health, 1994, 15, 107-132.

2 O. D. Boucher, P. Randall, P. Artaxo, C. Bretherton, G. Feingold, P. Forster, V.-M. Kerminen, Y. Kondon, H. Liao, U. Lohmann, P. Rasch, S. K. Satheesh, S. Sherwood, B. Stevens and X. Y. Zhang, Clouds and Aerosols. In: Climate Change 2013: The Physical Science Basis. Contribution of Working Group I to the Fifth Assessment Report of the Intergovernmental Panel on Climate Change, Cambridge University Press, Cambridge, 2013.

3 N. M. Donahue, S. A. Epstein, S. N. Pandis and a. L. Robinson, Atmos. Chem. Phys., 2011, 11, 3303-3318.

4 J. Seinfeld and S. Pandis, Atmospheric Chemistry and Physics. From Air Pollution to Climate Change, 2nd edn, 2006. 
5 T. F. Stocker, D. Qin, G. K. Plattner, M. Tignor, S. K. Allen, J. Boschung and A. Nauels, IPCC Climate Change 2013; The Physical Science Basis, 2013.

6 U. Lohmann and J. Feichter, Atmos. Chem. Phys. Discuss., 2004, 4, 7561-7614.

7 N. Li, C. Sioutas, A. Cho, D. Schmitz, C. Misra, J. Sempf, M. Wang, T. Oberley, J. Froines and A. Nel, Environ. Health Perspect., 2003, 111, 455-460.

8 J. Lelieveld, J. S. Evans, M. Fnais, D. Giannadaki and A. Pozzer, Nature, 2015, 525, 367-371.

9 F. Lavorini, C. J. Corrigan, P. J. Barnes, P. R. N. Dekhuijzen, M. L. Levy, S. Pedersen, N. Roche, W. Vincken and G. K. Crompton, Respir. Med., 2011, 105, 1099-1103.

10 J. Bell and S. Newman, Expert Opin. Drug Delivery, 2007, 4, 215-234.

11 B. Aggarwal and J. Gogtay, Expert Rev. Respir. Med., 2014, 8, 349-356.

12 A. R. Clark, Aerosol Sci. Technol., 1995, 22, 374-391.

13 K. Johnson, Adv. Drug Delivery Rev., 1997, 26, 3-15.

14 U. Pöschl, Angew. Chem., Int. Ed., 2005, 44, 7520-7540.

15 J. F. Pankow, Atmos. Environ., 1994, 28, 185-188.

16 M. Hallquist, J. C. Wenger, U. Baltensperger, Y. Rudich, D. Simpson, M. Claeys, J. Dommen, N. M. Donahue, C. George, A. H. Goldstein, J. F. Hamilton, H. Herrmann, T. Hoffmann, Y. Iinuma, M. Jang, M. E. Jenkin, J. L. Jimenez, A. Kiendler-Scharr, W. Maenhaut, G. McFiggans, T. F. Mentel, A. Monod, A. S. H. Prévôt, J. H. Seinfeld, J. D. Surratt, R. Szmigielski and J. Wildt, Atmos. Chem. Phys., 2009, 9, 5155-5236.

17 S. Martin, Chem. Rev., 2000, 100, 3403-3454.

18 S. Upadhyay, A. Parikh, P. Joshi and U. M. Upadhyay, J. Appl. Pharm. Sci., 2011, 01, 34-44.

19 V. Kundoor and R. N. Dalby, Pharm. Res., 2011, 28, 1895-1904.

20 Y. Guo, B. Laube and R. Dalby, Pharm. Res., 2005, 22, 1871-1878.

21 A. Haddrell, J. Davies, R. Miles, J. Reid, L. Dailey and D. Murnane, Int. J. Pharm., 2014, 463, 50-61.

22 H. Tong, C. Fitzgerald, P. Gallimore, M. Kalberer, M. Kuimova, P. Seville, A. Ward and F. Pope, Chem. Commun., 2014, 50, 15499-15502.

23 D. Broday and P. Georgopoulos, Aerosol Sci. Technol., 2001, 34, 144-159.

24 M. Y. Foo, Y.-S. Cheng, W.-C. Su and M. D. Donovan, J. Aerosol Med., 2007, 20, 495-508.

25 T. V. Vu, J. M. Delgado-Saborit and R. M. Harrison, Air Qual., Atmos. Health, 2015, 8, 429-440.

26 A. M. Booth, B. Murphy, I. Riipinen, C. J. Percival and D. O. Topping, Environ. Sci. Technol., 2014, 48, 9298-9305.

27 N. A. Hosny, C. Fitzgerald, C. Tong, M. Kalberer, M. K. Kuimova and F. D. Pope, Faraday Discuss., 2013, 165, 343-356.

28 N. A. Hosny, C. Fitzgerald, A. Vyšniauskas, A. Athanasiadis, T. Berkemeier, N. Uygur, U. Pöschl, M. Shiraiwa, M. Kalberer, F. D. Pope and M. K. Kuimova, Chem. Sci., 2016, 7, 1357-1367.

29 M. Kuimova, Phys. Chem. Chem. Phys., 2012, 14, 12671-12686. 30 T. Förster and G. Hoffman, Z. Phys. Chem., 1971, 75, 63-76.
31 N. Hosny, G. Mohamedi, P. Rademeyer, J. Owen, Y. Wu, M. Tang, R. Eckersley, E. Stride and M. Kuimova, Proc. Natl. Acad. Sci. U. S. A., 2013, 110, 9225-9230.

32 M. Haidekker and E. Theodorakis, J. Biol. Eng., 2010, 4, 11.

33 M. Dent, I. Lopez Duarte, C. Dickson, N. Geoghegan, J. Cooper, I. Gould, R. Krams, J. Bull, N. J. Brooks and M. K. Kuimova, Phys. Chem. Chem. Phys., 2015, 17, 18393-18402. 34 W. Akers, J. Biomech. Eng., 2004, 126, 340.

35 R. Power, D. L. Bones and J. P. Reid, in Optical Trapping and Optical Micromanipulation, ed. K. Dholakia and G. C. Spalding, 2012, vol. 8458, p. 845829.

36 L. Rkiouak, M. Tang, J. Camp, J. McGregor, I. Watson, R. Cox, M. Kalberer, A. Ward and F. Pope, Phys. Chem. Chem. Phys., 2014, 16, 11426-11434.

37 T. J. Harvey, E. C. Faria, A. Henderson, E. Gazi, A. D. Ward, N. W. Clarke, M. D. Brown, R. D. Snook and P. Gardner, J. Biomed. Opt., 2013, 13, 064004.

38 M. Bennet, P. Richardson, J. Arlt, A. McCarthy, G. Buller and A. Jones, Lab Chip, 2011, 11, 3821.

39 M. P. Longinotti and H. R. Corti, J. Phys. Chem. Ref. Data, 2008, 37, 1503.

40 A. J. Thompson, T. W. Herling, M. Kubánková, A. Vyšniauskas, T. P. J. Knowles and M. K. Kuimova, J. Phys. Chem. B, 2015, 119, 10170-10179.

41 A. Vyšniauskas, M. Balaz, H. L. Anderson and M. K. Kuimova, Phys. Chem. Chem. Phys., 2015, 17, 7548-7554.

42 eMC, Electron. Med. Compend., 2015.

43 S. Jones, M. King and A. Ward, Phys. Chem. Chem. Phys., 2013, 15, 20735.

44 S. Botchway, K. Scherer, S. Hook, C. Stubbs, E. Weston, R. Bisby and A. Parker, J. Microsc., 2015, 258, 68-78.

45 P. Barber, S. Ameer-Beg, J. Gilbey, L. Carlin, M. Keppler, T. Ng and B. Vojnovic, J. R. Soc., Interface, 2009, 6, S93-S105. 46 D. Marquardt, J. Soc. Ind. Appl. Math., 1963, 11, 431-441.

47 F. Fereidouni, A. Esposito, G. Blab and H. Gerritsent, J. Microsc., 2011, 244, 248-258.

48 M. Yaseen, S. Sakadžić, W. Wu, W. Becker, K. Kasischke and D. Boas, Biomed. Opt. Express, 2013, 4, 307-321.

49 B. Zobrist, V. Soonsin, B. Luo, U. Krieger, C. Marcolli, T. Peter and T. Koop, Phys. Chem. Chem. Phys., 2011, 13, 3514-3526.

50 R. M. Power and J. P. Reid, Rep. Prog. Phys., 2014, 77, 1-27.

51 H. J. Tong, J. P. Reid, D. L. Bones, B. P. Luo and U. K. Krieger, Atmos. Chem. Phys., 2011, 11, 4739-4754.

52 M. Laliberté, J. Chem. Eng. Data, 2007, 52, 321-335.

53 N. Labiris and M. Dolovich, Br. J. Clin. Pharmacol., 2003, 56, 588-599.

54 M. Hindle and P. Longest, J. Pharm. Pharmacol., 2012, 64, 1254-1263.

55 M. Shiraiwa, M. Ammann, T. Koop and U. Pöschl, Proc. Natl. Acad. Sci. U. S. A., 2011, 108, 11003-11008.

56 J. Patton and P. Byron, Nat. Rev. Drug Discovery, 2007, 6, 67-74. 57 C. Cothern and J. Smith, Environmental Radon. Environmental Science Research, Plenum Press, New York, vol. 35, 1987.

58 O. R. Hunt, A. D. Ward and M. D. King, RSC Adv., 2013, 3, 19448.

59 L. J. Moore, M. D. Summers and G. A. D. Ritchie, Phys. Chem. Chem. Phys., 2013, 15, 13489-13498. 